\title{
Identification of immune related cells and crucial genes in the peripheral blood of ankylosing spondylitis by integrated bioinformatics analysis
}

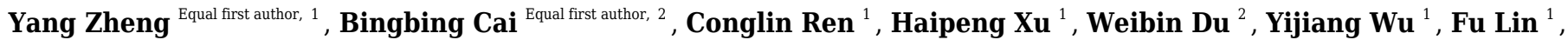 \\ Helou Zhang ${ }^{1}$, Renfu Quan ${ }^{\text {corresp. 1, 2, } 3}$ \\ ${ }^{1}$ Zhejiang Chinese Medical University, Hangzhou, China \\ 2 Department of Orthopedics, Hangzhou Xiaoshan Hospital of Traditional Chinese Medicine, Hangzhou, China \\ 3 Research Institute of Orthopedics, the Affiliated Jiangnan Hospital of Zhejiang Chinese Medical University, Hangzhou, China \\ Corresponding Author: Renfu Quan \\ Email address: quanrenfu@126.com
}

Background. Ankylosing spondylitis (AS) is a progressive rheumatic disease and studies reveal that the immune system is critical for the pathogenesis of AS. In the present study, various bioinformatics analysis methods were comprehensively applied, designed to identify potential key genes and inflammation states of AS.

Methods. The transcriptome profiles of GSE25101 and GSE73754 obtained from the Gene Expression Omnibus (GEO) database were merged for subsequent analyses. The differentially expressed genes (DEGs) were identified using the Bioconductor package Limma and threshold values. Functional enrichment and pathway enrichment analyses were performed using the clusterProfiler package and Gene Set Enrichment Analysis (GSEA). Next, protein-protein interaction (PPI) network of the identified DEGs was constructed by the online database, the Search Tool for the Retrieval of Interacting Genes (STRING), visualization and analysis were performed through Cytoscape software. Subsequently, we applied CIBERSORT algorithm to identify subpopulation proportions of immune cells in peripheral blood samples. Finally, we validated the hub genes with GSE18781 dataset. Samples were collected from patients to validate gene and protein expression using qRT-PCR and ELISA.

Results. A total of 334 DEGs were identified, including 182 upregulated and 152 downregulated DEGs, between AS patients and normal human controls, which were primarily involved in immune response, autophagy, and natural killer cell-mediated cytotoxicity. The most prominent module and candidate biomarkers were identified from the PPI network. Biomarkers were selected for validation and their expressions were significantly decreased in peripheral blood samples which was consistent with transcriptome sequencing results. Nine genes with AUC>0.70 were considered to be AS hub genes for ROC curve analysis, including GZMA, GZMK, PRF1, GNLY, NKG7, KLRB1, KLRD1, IL2RB and CD247. Furthermore, CIBERSORT results suggest that AS contained a higher proportion of CD8+ T cells, naive CD4+ T cells, neutrophils, and lower levels of gamma delta T cells compared with the normal controls.

Conclusion. In this study, we identified DEGs combined with their closely related biological functions and propose that granule-associated proteins and immune infiltration maybe involved in the progression of ankylosing spondylitis. These validated hub genes may provide new perspectives for understanding the molecular mechanisms of ankylosing spondylitis. 
1 Identification of immune related cells and crucial genes in the peripheral blood of

2 ankylosing spondylitis by integrated bioinformatics analysis

3

4

5

6

7

8

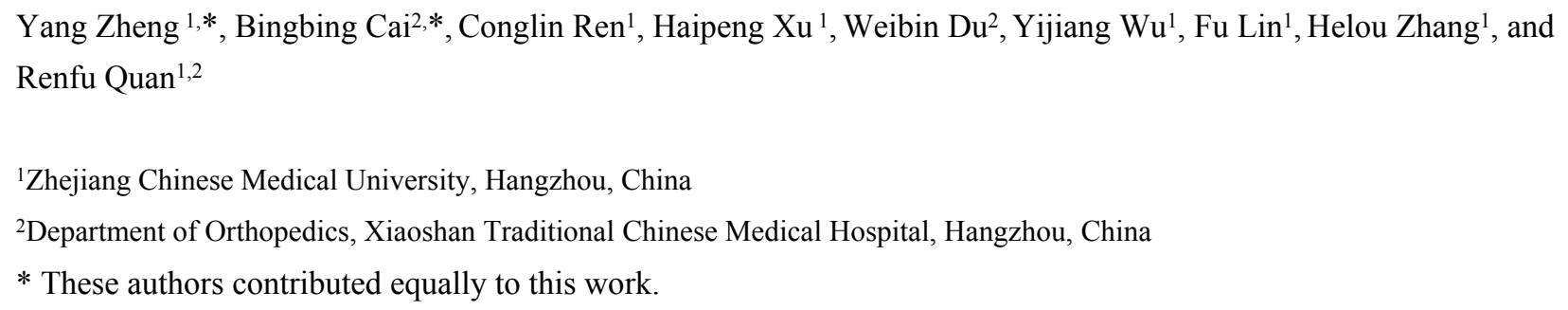

\section{ABSTRACT}

Background. Ankylosing spondylitis (AS) is a progressive rheumatic disease and studies reveal that the immune system is critical for the pathogenesis of AS. In the present study, various bioinformatics analysis methods were comprehensively applied, designed to identify potential key genes and inflammation states of AS.

Methods. The transcriptome profiles of GSE25101 and GSE73754 obtained from the Gene Expression Omnibus (GEO) database were merged for subsequent analyses. The differentially expressed genes (DEGs) were identified using the Bioconductor package Limma and threshold values. Functional enrichment and pathway enrichment analyses were performed using the clusterProfiler package and Gene Set Enrichment Analysis (GSEA). Next, protein-protein interaction (PPI) network of the identified DEGs was constructed by the online database, the Search Tool for the Retrieval of Interacting Genes (STRING), visualization and analysis were performed through Cytoscape software. Subsequently, we applied CIBERSORT algorithm to identify subpopulation proportions of immune cells in peripheral blood samples. Finally, we validated the hub genes with GSE18781 dataset. Samples were collected from patients to validate gene and protein expression using qRT-PCR and ELISA.

Results. A total of 334 DEGs were identified, including 182 upregulated and 152 downregulated DEGs, between AS patients and normal human controls, which were primarily involved in immune response, autophagy, and natural killer cell-mediated cytotoxicity. The most prominent module and candidate biomarkers were identified from the PPI network. Biomarkers were selected for validation and their expressions were significantly decreased in peripheral blood samples which was consistent with transcriptome sequencing results. Nine genes with AUC $>0.70$ were considered to be AS hub genes for ROC curve analysis, including GZMA, GZMK, PRF1, GNLY, NKG7, KLRB1, KLRD1, IL2RB and CD247. Furthermore, CIBERSORT results suggest that AS contained a higher proportion of CD8+ $\mathrm{T}$ cells, naive CD4+ T cells, neutrophils, and lower levels of gamma delta $\mathrm{T}$ cells compared with the normal 
controls.

Conclusion. In this study, we identified DEGs combined with their closely related biological functions and propose that granule-associated proteins and immune infiltration may be involved in the progression of ankylosing spondylitis. These validated hub genes may provide new perspectives for understanding the molecular mechanisms of ankylosing spondylitis.

\section{INTRODUCTION}

Ankylosing spondylitis (AS) is a chronic autoimmune inflammatory disease mainly involving the axial skeleton. The onset of this disease is predominantly seen in males before age 40 (Braun \& Sieper 2007; Chen et al. 2019; Smith 2015). It is estimated that its prevalence in the US is $0.9 \%$ to $1.4 \%$ of the adult population, which is similar to rheumatoid arthritis (Reveille et al. 2012). Its clinical manifestations and diagnostic points include chronic axial stiffness or inflammatory back pain, improvement with exercise, imaging data of the pelvis and spine, and serological examinations, especially for the HLA-B27 biomarker (Taurog et al. 2016). AS onset is associated with a combination of hereditary risks, the intestinal microbiome, and the immune response. However, a number of studies have reported that human leukocyte antigen B27 (HLAB27) is closely related to AS susceptibility. The genetic risk posed by HLA-B27 causes a predisposition toward AS and accounts for approximately $30 \%$ of cases, suggesting that other elements may also play a critical role in the development of this disease (Hu et al. 2020; Mohammadi et al. 2018).

Most studies have reported ankylosing spondylitis as an autoimmune disease marked by the activation of the immune system and subsequent chronic inflammation. These symptoms are confirmed by distinct inflammatory cell infiltration in the involved joints and by the therapeutic efficacy of NSAIDs and TNFa blockers (Duan et al. 2017; Vanaki et al. 2018). However, it is not fully understood how the immune system changes in AS and how these alterations meditate arthritis. Studying abnormal immune infiltrating cells may provide new strategies for treatment and diagnosis in light of the immune cell imbalance in AS.

Multiple gene expression profiling studies using microarray and bioinformatics analysis have been conducted to identify disease-associated biomarkers and pathways. These studies have attempted to highlight transcriptome differences between various phenotypes and stages of disease. The microarray technique in particular is a powerful tool for exploring gene regulation patterns and molecular mechanisms related to oncogenesis and progression of AS. However, these studies' results are limited or inconsistent due to significant variability between different projects or small sample sizes. Integrated bioinformatics approaches have been applied in biological research to assist in the development of more sensitive and effective diagnostic and therapeutic strategies. We downloaded the transcriptome datasets for AS patients and healthy controls from the Gene Expression Omnibus (GEO) database to identify key genes and the associated biological processes. These results may improve our understanding of AS development and help create new diagnostic and immunoregulatory therapeutic targets for treatment. 
80

81

82

83

84

85

86

87

88

89

90

91

92

93

94

95

96

97

98

99

100

101

102

103

104

105

106

107

108

109

110

111

112

113

114

115

116

117

118

\section{MATERIALS AND METHODS}

\section{Microarray data}

We used the GEO database (http://www.ncbi.nlm.nih.gov/geo) to obtain gene expression profiles for ankylosing spondylitis. A total of 36 healthy and 68 diseased specimens were collected from the gene expression profiles of GSE25101 and GSE73754. Additionally, the mRNA profiles of 25 healthy and 18 diseased specimens in the GSE18781 dataset were used to detect whether discovered hub genes had good diagnostic value for AS.

\section{Data normalization}

The series matrix for each expression dataset was also obtained from the GEO database. R software (version 4.0.1; https://www.r-project.org/) and Bioconductor packages (http://www.bioconductor.org/) were used for the bioinformatics analysis. First, the "affy" package in $\mathrm{R}$ was used to conduct normalization and background correction. Next, probe expression data were converted into gene expression matrices according to the platform annotation file. The average expression value was used for further analysis for various probes corresponding to one gene. Lastly, the "sva" $\mathrm{R}$ package was applied to eliminate differences between the 68 AS patient and 36 healthy control samples (Leek et al. 2012).

\section{Differentially expressed genes}

In $\mathrm{R}$ the limma package used to screen out differentially expressed genes (DEGs) based on the comparison of expression data between AS samples and normal samples with the selection criteria of adjusted $\mathrm{P}<0.05$ and $|\log \mathrm{FC}|>0.2$. We used the limma and pheatmap packages to create volcano plots and heatmaps for DEGs in RStudio (version:1.3.959) (Ritchie et al. 2015).

\section{Functional enrichment analysis for DEGs}

The Bioconductor package clusterProfiler (Yu et al. 2012) was used to evaluate DEGs' Gene Ontology (GO) and Kyoto Encyclopedia of Genes and Genomes (KEGG) pathway enrichment analysis. We found significant biological processes and pathways. GO terms and KEGG pathways with significant enrichment were identified using an adjusted $\mathrm{p}$ of $<0.05$. Gene Set Enrichment Analysis (GSEA) is a computational method for analyzing statistically significant and concordant differences between two groups (e.g., AS and the control) using a priori defined gene set (Subramanian et al. 2005). The expression values and phenotype labels in the GSE25101 and GSE73754 datasets were used to conduct GSEA analysis according to AS status (AS vs. non-AS) with the KEGG gene sets as a reference. We set the cut-off criteria as nominal $\mathrm{p}$ value $<0.01$ and false discovery rate (FDR) $<0.25$. The results were shown using the "ggplot2" package in R.

PPI network construction and analysis 
119 The STRING database (http://www.string-db.org/) was used to search for associations between known

120

121

122

123

124

125

126

127

128

129

130

131

132

133

134

135

136

137

138

139

140

141

142

143

144

145

146

147

148

149

150

151

152

153

154

155

and predicted proteins. This database is commonly used to evaluate PPI information by calculating their combined score. Cytoscape 3.5.0 (version 3.7.2; https://cytoscape.org/) was used to construct and visualize the results from the PPI network; the criteria was a combined score of $>0.4$ (Otasek et al. 2019). Cytoscape's plugin MCODE, which can find the most highly connected cluster in a complex network, was used to extract the most significant module in the PPI networks. CytoHubba (Chin et al. 2014) was utilized to calculate the degree centrality of protein nodes to search for hub genes using the Maximal Clique Centrality (MCC) method. Results were ranked based on MCC scores. These applications were used to identify key modules and hub genes in the PPI network with default parameters.

\section{Estimation of immune cell subtype distribution}

The CIBERSORT algorithm [13] was used to transform the normalized gene expression value into the component of peripheral blood immune cells with the LM22 reference signature matrix set at 1,000 permutations. We retained the CIBERSORT output with $\mathrm{p}$ value of $<0.05$ for subsequent analysis and calculated the percentage of immune cell subtypes in all samples. We applied CIBERSORT to characterize the immune cells in AS and control samples. In R we used bar and violin plots to depict the infiltration levels of immune cells from different subpopulations between the two groups.

\section{Validation of the hub genes by quantitative real-time PCR}

Peripheral blood samples from six AS patients and six patients without AS were collected for qRT-PCR validation to confirm the results of our bioinformatics analysis. The study protocol was approved by the Ethics Committee of Xiaoshan Traditional Chinese Medical Hospital, and all patients signed the informed consent. Mononuclear cells from peripheral blood were isolated by density gradient separation using standard operating procedures (STEMCELL Technologies Inc., Canada). Four milliliters of peripheral blood were collected in heparin sodium tubes prior to treatment. The blood was diluted with an equal amount of Dulbecco's phosphate buffered saline with 2\% fetal bovine serum (STEMCELL Technologies Inc., Canada). Samples were then layered on top of lymphoprep (STEMCELL Technologies Inc., Canada) and centrifuged at $800 \mathrm{x}$ g for 20 minutes at room temperature. The layer of mononuclear cells was washed twice with phosphate buffered saline. Total RNA from peripheral blood was extracted using a RNA-Quick Purification Kit (YISHAN BIOTECHNOLOGY, Shanghai, China). The concentration and purity of total RNAs were measured with Nanodrop One (Thermo Scientific Inc., USA), and only RNA samples with Abs $260 \mathrm{~nm} / \mathrm{Abs} 280 \mathrm{~nm}$ ratio $>1.8$ were used. Total RNAs were reverse transcribed to cDNA using a RevertAid First Strand cDNA Synthesis Kit (Thermo Scientific Inc., USA), and qRT-PCR was conducted using the PowerUp ${ }^{\text {TM }}$ SYBR ${ }^{\text {TM }}$ Green Mix (Thermo Scientific Inc., USA) on the Applied Biosystems $^{\mathrm{TM}} 7500$ (Thermo Scientific Inc., USA). We used GAPDH as an internal control. All primers used in this study are shown in Table 1 . The relative mRNA expression was normalized and calculated using the comparative $\mathrm{Ct}$ method $(2-\Delta \Delta \mathrm{Ct})$. The data were presented as the fold change of expression

Peer] reviewing PDF | (2021:02:57983:2:1:NEW 31 Jul 2021) 
156 relative to normal tissues. One-way analysis of variance was conducted for comparison, and $\mathrm{P}<0.05$

157 indicated statistically significant differences.

158

159

160

161

162

163

164

165

166

167

168

169

170

171

172

173

174

175

176

177

178

179

180

181

182

183

184

185

186

187

188

189

190

191

192

193

\section{Validation of hub genes with the GEO database}

Genes in the PPI network identified by the MCODE plug-in were selected as key candidate genes. We applied ROC analysis to evaluate the prediction efficacy of these candidate genes and calculated the area under curve (AUC) with RStudio. The genes with an AUC of $>0.7$ as well as a p-value of $<0.05$ were considered to be AS hub genes.

\section{Enzyme-linked immunosorbent assay}

Serum was obtained from three AS patients and three patients without AS. The serum level of GZMA, GNLY, and PRF1 were analyzed with a Human Granulysin ELISA Kit (EK1280), Human Granzyme A ELISA Kit (EK1162), and Human PRF1 ELISA Kit (MM-0254H2) according to the manufacturers' protocols. The absorbance was determined to be $450 \mathrm{~nm}$ by EnSpire (PerkinElmer, USA)

\section{RESULTS}

\section{Identification of DEGs}

Raw data were read, corrected, and normalized. We obtained 334 DEGs including 182 upregulated and 152 downregulated genes after batch correction and normalization of the integrated dataset (GSE25101 and GSE73754) (Table S1). All DEGs were shown using a volcano plot (Fig.1A) and the expression of the top 50-upregulated and downregulated genes was shown with a heatmap (Fig.1B).

\section{GO and KEGG enrichment analysis of DEGs}

Functional enrichment analyses were performed using the clusterProfiler package for further insight into the biological functions and pathways related to DEGs. For the GO analysis (Table 2 and Fig. 2A), DEGs were primarily enriched in I-kappaB kinase/NF-kappaB signaling, immune cell related processes, autophagy, process utilizing autophagic mechanism, positive regulation of cytokine production, and response to molecule of bacterial origin. KEGG pathway analysis showed that DEGs primarily participated in Phagosome, Leishmaniasis, Hematopoietic cell lineage, Natural killer cell-mediated cytotoxicity, Necroptosis, Apoptosis, Graft-versus-host disease, Acute myeloid leukemia, and Th1 and Th2 cell differentiation (Table 3 and Fig.2B).

\section{GSEA analysis}

We used GSEA to perform a pathway enrichment analysis, defined by KEGG gene sets, to further explore the biological function of DEGs. According to the pre-set threshold of analysis results, complement and coagulation cascades, leukocyte transendothelial migration, osteoclast differentiation, and autophagyanimal gene sets were significantly upregulated in AS samples. Results are detailed in Fig. 3.

Peer] reviewing PDF | (2021:02:57983:2:1:NEW 31 Jul 2021) 
194

195

196

197

198

199

200

201

202

203

204

205

206

207

208

209

210

211

212

213

214

215

216

217

218

219

220

221

222

223

224

225

226

227

228

229

230

231

232

233

234

\section{PPI network construction and analysis}

To further explore the relationships between DEGs at the protein level, the PPI network of DEGs was constructed by online STRING database with the predefined criteria, and visualized using Cytoscape. Upregulated and downregulated nodes (DEGs) were labeled with red and blue, respectively (Fig.4A). The diameters of the nodes indicated their degree of connectivity. The most significant module with the highest score (9.000) detected by MCODE analysis is shown in Fig. 4B. It consists of 23 genes and 99 edges. The top 10 critical genes were selected by calculating MCC scores using the cytoHubba plugin; these genes are presented in Fig. 4C. All critical genes were downregulated in AS samples compared to control samples. The 10 candidate hub nodes were all contained in the module mentioned above, which implied that the module may be a good representation of critical biological characteristics. The 10 nodes were thus defined as the key nodes in the PPI network. The KEGG of these DEGs was analyzed using the clusterProfiler package. Our results showed that these genes were mainly enriched in the functions of natural killer cell mediated cytotoxicity, graft-versus-host disease, Th1 and Th2 cell differentiation, and Th17 cell differentiation (Table 4).

\section{Distribution pattern of immune cell subtype}

The overall distribution of different immune subsets in all samples was shown in a histogram (Fig. 5A). Different colors and heights represent various types and percentages of immune cells in the sample, and the sum of the proportion of various immune cells is 1 . CD8 $+\mathrm{T}$ cells, naive CD4+ T cells, gamma delta $\mathrm{T}$ cells, and neutrophils were the primary infiltrating cells. There are individual differences in the proportion of immune cells between the two groups. Clustering analysis of infiltrating immune cells in AS and control samples is a vital for identifying immunopathogenesis (Fig. 5B). The different immune cell subsets were weakly-to-moderately correlated. The correlation of NK cells and neutrophils was 0.5 , the correlation between neutrophils and CD8+ T cells was 0.66 (Fig. 5C). Compared with healthy controls, the proportion of CD8 $+\mathrm{T}$ cells $(\mathrm{p}=0.007)$, naive $\mathrm{CD} 4+\mathrm{T}$ cells $(\mathrm{p}=0.041)$, and neutrophils $(\mathrm{p}=0.007)$ was higher in AS samples, while the fraction of gamma delta T cells $(p=0.012)$ was relatively lower (Fig. $5 \mathrm{D})$.

\section{Validation of candidate biomarkers}

The qRT-PCR results showed a significant downregulation in the expression of 10 hub genes (GZMK, GNLY, KLRD1, GZMB, PRF1, IL2RB, KLRB1, NKG7, CD247, and GZMA) obtained from cytoHubba analysis. These results were consistent with the results of the microarray hybridization, suggesting that the results were convincing (Fig. 6). ELISA analysis suggested that protein expression levels of GZMA, GNLY, and PRF1 in serum were significantly decreased in the AS group compared to the control group (Fig. 7).

\section{Receiver operating characteristic (ROC) curve analysis}

The validation dataset (GSE18781) was obtained from the GEO database. We performed ROC curve analysis using RStudio to further verify the reliability of these 10 candidate genes in patients' peripheral blood. Nine of the ten genes (GZMA, GZMK, NKG7, PRF1, GNLY, KLRD1, KLRB1, IL2RB, CD247) with an AUC of more than 0.70 were considered hub genes, indicating that they may be able to diagnose 
235

236

\section{Discussion}

AS patients with excellent specificity and sensitivity (Fig. 8).

AS is a chronic inflammatory rheumatic disease characterized by two major features: chronic inflammation and progressive ankylosis in the axial skeleton. This disease leads to a clinical picture that includes inflammatory back pain, asymmetrical peripheral oligoarthritis, chronic inflammatory bowel disease (IBD), progressive stiffness, and loss of spinal mobility (Appel et al. 2006; Blair 2019; Braun \& Sieper 2007). The interaction between host genetics, the intestinal microbiome, and the immune response is strongly related to AS pathogenesis. (Qiao et al. 2019; Wang et al. 2016; Yang et al. 2016). Previous bioinformatics research found that some immune-associated pathways like T-cell receptor signaling pathway, natural killer cell mediated cytotoxicity were significantly enriched in AS patients (Chen et al. $2012 ; \mathrm{Xu}$ et al. 2019). And one bioinformatics study revealed that some TNF and interleukin (IL) related factors were detected as DEGs, such as IL2RB, IL17RB and IL17RD, which might be associated with in the progression of AS (Zhao et al. 2015). These studies have demonstrated the importance of immune cells in determining the pathogenesis of AS. Given the progressive and often disabling nature of AS, it is important to identify the novel molecular targets and potential mechanisms of AS to provide underlying biomarkers or therapeutic approaches. We screened out 334 DEGs, including 182 upregulated and 152 downregulated genes, by comparing the differences in gene expression profiles of peripheral blood in AS patients and healthy controls. Functional enrichment analysis was performed to further explore the regulatory roles of DEGs in AS. We constructed a PPI network containing DEGs in the STRING database. Cytoscape software was used to visualize interactions between DEGs. The MCODE plug-in and Cytohubba were used to screen the most significant module and hub genes in the PPI network.

The results of enrichment analysis showed that the module and DEGs were significantly correlated with immune-related functions and inflammatory signaling, such as the activation and regulation of IkappaB kinase/NF-kappaB signaling, neutrophil activation, Th1 and Th2 cell differentiation, and Th17 cell differentiation. T helper (Th) cells are divided into Th1 and Th2 subsets according to their cytokine production profiles (Abbas et al. 1996). Previous studies found that Th1 and Th2 chemoattractants played a cooperative role in the development of AS. Th1 and Th2 chemokine levels decreased under etanercept, a TNF- $\alpha$ blocker, to improve the activity and functional capacity of AS patients (Ergin et al. 2011; Wang et al. 2016; Zhang et al. 2018). Animal experiments indicated that Micheliolide, a sesquiterpene lactone involved in alleviating the inflammatory response, maintained the balance of Th1/Th2 by regulating NF$\kappa \mathrm{B}$ signaling (Tian et al. 2020). Th17 cells were shown to be involved in AS (Xueyi et al. 2013). T-helper 17 (Th17) cells are a subset of CD4+ T cells and were found to be related to the pathogenesis of autoimmune diseases and inflammatory diseases such as AS, rheumatoid arthritis (RA), and inflammatory bowel disease (IBD) (Hammitzsch et al. 2018). Th17 cells produce proinflammatory cytokine and interleukin-17 (IL-17), and their differentiation is regulated by the presence of IL-23 (Hajialilo et al. 2019). In addition, antibodies targeting the IL-23/ IL-17 axis have demonstrated efficacy; a genome-wide association study of AS found that IL-23 and IL-1 cytokine pathways play a role in disease susceptibility(Reveille et al. 2010). This effect plays a key role for T helper cell type 17 (T17) responses 
in the pathogenesis of AS (Baeten et al. 2015; Karaderi et al. 2009). These findings imply that T helper cell responses are instrumental for the pathogenesis of AS and deserve more attention.

We analyzed the proportion of blood immune cell subsets using CIBERSORT, a transcriptome deconvolution algorithm, to better understand the role of leukocyte infiltration and the inflammatory response in the pathogenesis and development of AS. Of the 22 major immune cell types, we found significant differences in the composition of immune cells between AS and healthy controls. These differences include an increase in the proportion of CD8 $\mathrm{T}$ cells, naive CD4+ T cells, neutrophils, and the lower portion of Gamma Delta T cells. These differences were also significantly associated with AS. Our results were consistent with previously reported variations in the abundancies of various cells in AS (Shamji et al. 2008; Zhang et al. 2009).

GO and GSEA enrichment analysis indicated that autophagy may play a vital role in the pathological development of AS. Autophagy is a cellular lysosomal degradation pathway to remove pathogens and dead cells. Autophagy also inhibits inflammasome activation and secretion that is important for cellular homeostasis (Ma et al. 2019; Zhai et al. 2018). It is well known that autophagy plays a potentially key role in immune cells' induction and regulation of inflammatory responses. Therefore, dysfunction in this process may lead to inflammation, such as multiple sclerosis and rheumatoid arthritis (Sutton et al. 2006). Ciccia et al. (2014) found that HLA-B27 misfolding occurs in AS patients' guts and is accompanied by autophagy activation rather than an unfolded protein response. A recent study illustrated the lower expression of autophagy-related genes and defective autophagy activity in AS patients' peripheral blood (Park et al. 2017). This research suggested that autophagy plays a role in AS pathogenesis.

Finally, we validated these crucial genes in PPI network by performing qRT-PCR and ELISA assay. As a result, 10 genes with $p$-value $<0.01$ were successfully validated for their low expression in AS patients, including granzyme family (GZMA, GZMB and GZMK), perforin 1 (PRF1), granule proteins (GNLY and NKG7), killer cell lectin like receptor (KLRB1 and KLRD1), and T-cell signaling genes (IL2RB and CD247). And 9 genes validated by ROC curve analysis with p-value $<0.05$ as well as AUC $>$ 0.70 showed potential diagnostic value for AS, and thus were considered as hub genes of AS, including GZMA, GZMK, PRF1, GNLY, NKG7, KLRB1, KLRD1, IL2RB and CD247.

Notably, many crucial genes expressed at very low levels in AS patients were linked to the lymphoid lineage, especially cytotoxic lymphocytes including cytotoxic T lymphocytes (CTLs) and natural killer (NK) cells. Cytotoxic T lymphocytes (CTLs) and natural killer (NK) cells are characterized by potent toxins secretion, including granule proteins, perforins, and granulysins stored in the secretory granules (SGs) of CTLs and NK cells (Anthony et al. 2010). They employ SGs to cleave and activate effector molecules within the target cell through granule exocytosis (Voskoboinik et al. 2015).

The KLRD1 and KLRB1 protein, expressed by NK cell, are classified as a type II membrane protein and may be involved in the regulation of NK cell function. A recent bioinformatics found KLRD1 and CD247 DEGs were significantly enriched in natural killer cell mediated cytotoxicity, regulation of immune response, which was consistent with our findings (Fan et al. 2019). IL2RB, an interleukin 2 receptor, is involved in T cell-mediated immune responses, which have been proved related to AS in previous study and a recent clinical study found significant associations with the presence of peripheral arthritis at AS onset in SNPs of IL2RB gene (Polo et al. 2019; Zhu et al. 2013). 
The granzyme family is a class of homologous serine proteases, mainly expressed by cytotoxic $\mathrm{T}$ lymphocytes (CTLs) and natural killer (NK) cells (Fehniger et al. 2007; Pardo et al. 2002; Shah et al. 2011). To date, five various human granzymes (GZMA, GZMB, GZMH, GZMK, and GZMM) have already been identified according to the primary substrate specificity (van Daalen et al. 2020). In addition, perforin and granzyme are tightly packed in the core of SGs and jointly mediate target cell apoptosis once SGs conjugated to a target cell (Trapani et al. 2013). Granulysin, encoded by GNLY, is also known as NKG5 and is similar to granzymes. It presents in SGs, diffuses into target cells through perforin pores and can induce the recruitment and activation of antigen-presenting cells (APCs), facilitating the generation of an immune response (Clayberger \& Krensky 2003; Tewary et al. 2010). Increasing evidence indicates that the abnormal expression of GZM family, granule proteins (NKG5 and NKG7), and perforin 1 (PRF1) is involved in a number of autoimmune diseases, including ankylosing spondylitis, rheumatoid arthritis (RA), hypersensitive pneumonitis, celiac disease, and scleroderma (Anthony et al. 2010; Prager et al. 2019). A previous study indicated the importance of PRF1 in immune response and immune regulation related functions in AS and highlighted its significance of common markers for rheumatic diseases (Wang et al. 2015). In addition, a recent cohort study confirmed that GZMA and PRF1 in AS and RA patients' peripheral blood were significantly downregulated compared to the controls, especially for male AS patients. On the other hand, GZMA and GZMB in the synovial fluid (SF) of AS patients was significantly higher than in the serum, while PRF1 expression in SF was further downregulated (Gracey et al. 2020). Additional research should be conducted to address the potential roles of granule proteins secreted by SGs in the pathogenesis of ankylosing spondylitis.

\section{CONCLUSIONS}

In summary, we applied a comprehensive bioinformatics method to show that immune cell infiltration and granule-associated proteins were important in the pathogenesis of ankylosing spondylitis. The hub genes we identified may serve as potential biomarkers for AS. However, further experiments are required to support our conclusions.

\section{ADDITIONAL INFORMATION AND DECLARATIONS}

\section{Funding}

This study was supported by the National Natural Science Foundation of China (NO. 81904053); Zhejiang Provincial Science and Technology Program (NO. 2020KY797), Hangzhou City Science and Technology Program (No. 20171226Y96), Zhejiang Chinese Medical University (NO. ZYX2018008). The funders had no role in study design, data collection and analysis, decision to publish, or preparation of the manuscript.

\section{Grant Disclosures}

The following grant information was disclosed by the authors:

National Natural Science Foundation of China (NO. 81904053). 
Zhejiang Provincial Science and Technology Program (NO. 2020KY797).

Hangzhou City Science and Technology Program (No. 20171226Y96).

Zhejiang Chinese Medical University (NO. ZYX2018008).

\section{Author Contributions}

- Yang Zheng and Bingbing Cai performed the experiments, analyzed the data, and approved the final draft.

- Renfu Quan and Yang Zheng conceived and designed the experiments, authored or reviewed drafts of the paper, and approved the final draft.

- Bingbing Cai and $\mathrm{Fu}$ Lin analyzed the data, prepared figures and tables, and approved the final draft.

- Weibin Du and Haipeng Xu performed the experiments, authored or reviewed drafts of the paper, and approved the final draft.

- Yijiang $\mathrm{Wu}$ and Conglin Ren analyzed the data, prepared figures and tables, and approved the final draft.

\section{Data Availability}

The following information was supplied regarding data availability: Data is available at NCBI GEO: GSE25101, GSE73754, GSE18781. The code is available as Supplementary Files.

\section{REFERENCES}

Abbas AK, Murphy KM, and Sher A. 1996. Functional diversity of helper T lymphocytes. Nature 383:787-793. 10.1038/383787a0

Anthony DA, Andrews DM, Watt SV, Trapani JA, and Smyth MJ. 2010. Functional dissection of the granzyme family: cell death and inflammation. Immunol Rev 235:73-92. 10.1111/j.0105-2896.2010.00907.x

Appel H, Kuhne M, Spiekermann S, Ebhardt H, Grozdanovic Z, Köhler D, Dreimann M, Hempfing A, Rudwaleit M, Stein H, Metz-Stavenhagen P, Sieper J, and Loddenkemper C. 2006. Immunohistologic analysis of zygapophyseal joints in patients with ankylosing spondylitis. Arthritis Rheum 54:2845-2851. $10.1002 /$ art.22060

Baeten D, Sieper J, Braun J, Baraliakos X, Dougados M, Emery P, Deodhar A, Porter B, Martin R, Andersson M, Mpofu S, and Richards HB. 2015. Secukinumab, an Interleukin-17A Inhibitor, in Ankylosing Spondylitis. N Engl J Med 373:2534-2548. 10.1056/NEJMoa1505066

Blair HA. 2019. Secukinumab: A Review in Ankylosing Spondylitis. Drugs 79:433-443. 10.1007/s40265-01901075-3

Braun J, and Sieper J. 2007. Ankylosing spondylitis. Lancet 369:1379-1390. 10.1016/s0140-6736(07)60635-7

Chen C, Rong T, Li Z, and Shen J. 2019. Noncoding RNAs Involved in the Pathogenesis of Ankylosing Spondylitis. Biomed Res Int 2019:6920281. 10.1155/2019/6920281

Chen K, Zhao Y, Chen Y, Wang C, Chen Z, Bai Y, Zhu X, and Li M. 2012 A sub-pathway based method to identify candidate agents for Ankylosing Spondylitis. Molecules 17:12460-12468. 10.3390/molecules 171012460 
Chin CH, Chen SH, Wu HH, Ho CW, Ko MT, and Lin CY. 2014. cytoHubba: identifying hub objects and subnetworks from complex interactome. BMC Syst Biol 8 Suppl 4:S11. 10.1186/1752-0509-8-s4-s11

Clayberger C, and Krensky AM. 2003. Granulysin. Curr Opin Immunol 15:560-565. 10.1016/s09527915(03)00097-9

Duan Z, Gui Y, Li C, Lin J, Gober HJ, Qin J, Li D, and Wang L. 2017. The immune dysfunction in ankylosing spondylitis patients. Biosci Trends 11:69-76. 10.5582/bst.2016.01171

Ergin A, Syrbe U, Scheer R, Thiel A, Adam T, Büssow K, Duchmann R, Zeitz M, and Sieper J. 2011. Impaired peripheral Th1 CD4+ $\mathrm{T}$ cell response to Escherichia coli proteins in patients with Crohn's disease and ankylosing spondylitis. J Clin Immunol 31:998-1009. 10.1007/s10875-011-9575-x

Fan X, Qi B, Ma L, and Ma F. 2019. Screening of underlying genetic biomarkers for ankylosing spondylitis. Mol Med Rep 19:5263-5274. 10.3892/mmr.2019.10188

Fehniger TA, Cai SF, Cao X, Bredemeyer AJ, Presti RM, French AR, and Ley TJ. 2007. Acquisition of murine NK cell cytotoxicity requires the translation of a pre-existing pool of granzyme B and perforin mRNAs. Immunity 26:798-811. 10.1016/j.immuni.2007.04.010

Gracey E, Yao Y, Qaiyum Z, Lim M, Tang M, and Inman RD. 2020. Altered Cytotoxicity Profile of CD8+ T Cells in Ankylosing Spondylitis. Arthritis Rheumatol 72:428-434. 10.1002/art.41129

Hajialilo M, Dolati S, Abdolmohammadi-Vahid S, Ahmadi M, Kamrani A, Eghbal-Fard S, Ghassembaglou A, Valizadeh A, Shenas MHM, Aghebati-Maleki L, Kafil HS, Mehdizadeh A, and Yousefi M. 2019. Nanocurcumin: A novel strategy in treating ankylosing spondylitis by modulating Th17 cells frequency and function. $J$ Cell Biochem. 10.1002/jcb.28488

Hammitzsch A, Chen L, de Wit J, Al-Mossawi MH, Ridley A, Sekine T, Simone D, Doig K, Skapenko A, and Bowness P. 2018. Inhibiting ex-vivo Th17 responses in Ankylosing Spondylitis by targeting Janus kinases. Sci Rep 8:15645. 10.1038/s41598-018-34026-1

Hu J, Ren W, Qiu W, Lv J, Zhang C, Xu C, Du W, Wu M, Wang J, and Quan R. 2020. Generation of induced pluripotent stem cell line (XDCMHi001-A) from an Ankylosing spondylitis patient with JAK2 mutation. Stem Cell Res 45:101788. 10.1016/j.scr.2020.101788

Karaderi T, Harvey D, Farrar C, Appleton LH, Stone MA, Sturrock RD, Brown MA, Wordsworth P, and Pointon JJ. 2009. Association between the interleukin 23 receptor and ankylosing spondylitis is confirmed by a new UK case-control study and meta-analysis of published series. Rheumatology (Oxford) 48:386-389. 10.1093/rheumatology/ken501

Leek JT, Johnson WE, Parker HS, Jaffe AE, and Storey JD. 2012. The sva package for removing batch effects and other unwanted variation in high-throughput experiments. Bioinformatics 28:882-883. 10.1093/bioinformatics/bts034

Ma C, Wen B, Zhang Q, Shao PP, Gu W, Qu K, Shi Y, and Wang B. 2019. Emodin induces apoptosis and autophagy of fibroblasts obtained from patient with ankylosing spondylitis. Drug Des Devel Ther 13:601-609. $10.2147 /$ dddt.S182087

Mohammadi H, Hemmatzadeh M, Babaie F, Gowhari Shabgah A, Azizi G, Hosseini F, Majidi J, and Baradaran B. 2018. MicroRNA implications in the etiopathogenesis of ankylosing spondylitis. $J$ Cell Physiol 233:5564-5573. 10.1002/jcp.26500

Otasek D, Morris JH, Bouças J, Pico AR, and Demchak B. 2019. Cytoscape Automation: empowering workflow-based network analysis. Genome Biol 20:185. 10.1186/s13059-019-1758-4 
Pardo J, Balkow S, Anel A, and Simon MM. 2002. The differential contribution of granzyme A and granzyme B in cytotoxic $\mathrm{T}$ lymphocyte-mediated apoptosis is determined by the quality of target cells. Eur J Immunol 32:1980-1985. 10.1002/1521-4141(200207)32:7<1980::Aid-immu1980>3.0.Co;2-z

Park MC, Kim HW, Lee SW, Song JJ, and Park YB. 2017. Defective autophagy activity and its association with spinal damage in patients with ankylosing spondylitis. Joint Bone Spine 84:583-587. 10.1016/j.jbspin.2016.09.005

Polo YLBJ, Szczypiorska M, Bartolomé N, Campos J, Flores-Robles BJ, Sanz J, Fernández-Espartero C, Clavaguera T, Andrus RF, Artieda M, Tejedor D, Martínez A, Mulero J, Isasi CM, Andréu JL, and Sánchez A. 2019. Clinical and genetic characteristics of ankylosing spondylitis patients with peripheral arthritis at disease onset. Clin Exp Rheumatol 37:215-221.

Prager I, Liesche C, van Ooijen H, Urlaub D, Verron Q, Sandström N, Fasbender F, Claus M, Eils R, Beaudouin J, Önfelt B, and Watzl C. 2019. NK cells switch from granzyme B to death receptor-mediated cytotoxicity during serial killing. J Exp Med 216:2113-2127. 10.1084/jem.20181454

Qiao M, Qian BP, Qiu Y, Mao SH, and Wang YH. 2019. Radiologic and Pathological Investigation of Pseudarthrosis in Ankylosing Spondylitis: Distinguishing Between Inflammatory and Traumatic Etiology. $J$ Rheumatol 46:259-265. 10.3899/jrheum.171249

Reveille JD, Sims AM, Danoy P, Evans DM, Leo P, Pointon JJ, Jin R, Zhou X, Bradbury LA, Appleton LH, Davis JC, Diekman L, Doan T, Dowling A, Duan R, Duncan EL, Farrar C, Hadler J, Harvey D, Karaderi T, Mogg R, Pomeroy E, Pryce K, Taylor J, Savage L, Deloukas P, Kumanduri V, Peltonen L, Ring SM, Whittaker P, Glazov E, Thomas GP, Maksymowych WP, Inman RD, Ward MM, Stone MA, Weisman MH, Wordsworth BP, and Brown MA. 2010. Genome-wide association study of ankylosing spondylitis identifies non-MHC susceptibility loci. Nat Genet 42:123-127. 10.1038/ng.513

Reveille JD, Witter JP, and Weisman MH. 2012. Prevalence of axial spondylarthritis in the United States: estimates from a cross-sectional survey. Arthritis Care Res (Hoboken) 64:905-910. 10.1002/acr.21621

Ritchie ME, Phipson B, Wu D, Hu Y, Law CW, Shi W, and Smyth GK. 2015. limma powers differential expression analyses for RNA-sequencing and microarray studies. Nucleic Acids Res 43:e47. 10.1093/nar/gkv007

Shah D, Kiran R, Wanchu A, and Bhatnagar A. 2011. Soluble granzyme B and cytotoxic T lymphocyte activity in the pathogenesis of systemic lupus erythematosus. Cell Immunol 269:16-21. 10.1016/j.cellimm.2011.03.004

Shamji MF, Bafaquh M, and Tsai E. 2008. The pathogenesis of ankylosing spondylitis. Neurosurg Focus 24:E3. $10.3171 /$ foc $/ 2008 / 24 / 1 / \mathrm{e} 3$

Smith JA. 2015. Update on ankylosing spondylitis: current concepts in pathogenesis. Curr Allergy Asthma Rep 15:489. 10.1007/s11882-014-0489-6

Subramanian A, Tamayo P, Mootha VK, Mukherjee S, Ebert BL, Gillette MA, Paulovich A, Pomeroy SL, Golub TR, Lander ES, and Mesirov JP. 2005. Gene set enrichment analysis: a knowledge-based approach for interpreting genome-wide expression profiles. Proc Natl Acad Sci $U$ S A 102:15545-15550. 10.1073/pnas.0506580102

Sutton C, Brereton C, Keogh B, Mills KH, and Lavelle EC. 2006. A crucial role for interleukin (IL)-1 in the induction of IL-17-producing T cells that mediate autoimmune encephalomyelitis. $J$ Exp Med 203:1685-1691. 10.1084/jem.20060285

Taurog JD, Chhabra A, and Colbert RA. 2016. Ankylosing Spondylitis and Axial Spondyloarthritis. $N$ Engl $J$ 
Med 374:2563-2574. 10.1056/NEJMra1406182

Tewary P, Yang D, de la Rosa G, Li Y, Finn MW, Krensky AM, Clayberger C, and Oppenheim JJ. 2010. Granulysin activates antigen-presenting cells through TLR4 and acts as an immune alarmin. Blood 116:34653474. 10.1182/blood-2010-03-273953

Tian ZG, Yao M, and Chen J. 2020. Micheliolide alleviates ankylosing spondylitis (AS) by suppressing the activation of the NLRP3 inflammasome and maintaining the balance of Th1/Th2 via regulating the NF- $\mathrm{KB}$ signaling pathway. Ann Transl Med 8:991. 10.21037/atm-20-4987

Trapani JA, Thia KY, Andrews M, Davis ID, Gedye C, Parente P, Svobodova S, Chia J, Browne K, Campbell IG, Phillips WA, Voskoboinik I, and Cebon JS. 2013. Human perforin mutations and susceptibility to multiple primary cancers. Oncoimmunology 2:e24185. 10.4161/onci.24185

van Daalen KR, Reijneveld JF, and Bovenschen N. 2020. Modulation of Inflammation by Extracellular Granzyme A. Front Immunol 11:931. 10.3389/fimmu.2020.00931

Vanaki N, Aslani S, Jamshidi A, and Mahmoudi M. 2018. Role of innate immune system in the pathogenesis of ankylosing spondylitis. Biomed Pharmacother 105:130-143. 10.1016/j.biopha.2018.05.097

Voskoboinik I, Whisstock JC, and Trapani JA. 2015. Perforin and granzymes: function, dysfunction and human pathology. Nat Rev Immunol 15:388-400. 10.1038/nri3839

Wang J, Zhao Q, Wang G, Yang C, Xu Y, Li Y, and Yang P. 2016. Circulating levels of Th1 and Th2 chemokines in patients with ankylosing spondylitis. Cytokine 81:10-14. 10.1016/j.cyto.2016.01.012

Wang L, Wu LF, Lu X, Mo XB, Tang ZX, Lei SF, and Deng FY. 2015. Integrated Analyses of Gene Expression Profiles Digs out Common Markers for Rheumatic Diseases. PLoS One 10:e137522. 10.1371/journal.pone.0137522

Xu Z, Zhou X, Li H, Chen Q, and Chen G. 2019. Identification of the key genes and long non-coding RNAs in ankylosing spondylitis using RNA sequencing. Int J Mol Med 43:1179-1192. 10.3892/ijmm.2018.4038

Xueyi L, Lina C, Zhenbiao W, Qing H, Qiang L, and Zhu P. 2013. Levels of circulating Th17 cells and regulatory $\mathrm{T}$ cells in ankylosing spondylitis patients with an inadequate response to anti-TNF- $\alpha$ therapy. $J$ Clin Immunol 33:151-161. 10.1007/s10875-012-9774-0

Yang C, Ding P, Wang Q, Zhang L, Zhang X, Zhao J, Xu E, Wang N, Chen J, Yang G, Hu W, and Zhou X. 2016. Inhibition of Complement Retards Ankylosing Spondylitis Progression. Sci Rep 6:34643. $10.1038 /$ srep34643

Yu G, Wang LG, Han Y, and He QY. 2012. clusterProfiler: an R package for comparing biological themes among gene clusters. Omics 16:284-287. 10.1089/omi.2011.0118

Zhai Y, Lin P, Feng Z, Lu H, Han Q, Chen J, Zhang Y, He Q, Nan G, Luo X, Wang B, Feng F, Liu F, Chen Z, and Zhu P. 2018. TNFAIP3-DEPTOR complex regulates inflammasome secretion through autophagy in ankylosing spondylitis monocytes. Autophagy 14:1629-1643. 10.1080/15548627.2018.1458804

Zhang CL, Li YC, Wu JW, and Zhu BL. 2018. Expression and function of peripheral blood miRNA16a in patients with ankylosing spondylitis. Eur Rev Med Pharmacol Sci 22:5106-5113. 10.26355/eurrev_201808_15704

Zhang L, Jarvis LB, Baek HJ, and Gaston JS. 2009. Regulatory IL4+CD8+ T cells in patients with ankylosing spondylitis and healthy controls. Ann Rheum Dis 68:1345-1351. 10.1136/ard.2008.088120

Zhao H, Wang D, Fu D, and Xue L. 2015. Predicting the potential ankylosing spondylitis-related genes utilizing bioinformatics approaches. Rheumatol Int 35:973-979. 10.1007/s00296-014-3178-9 
519 Zhu ZQ, Tang JS, and Cao XJ. 2013. Transcriptome network analysis reveals potential candidate genes for 520 ankylosing spondylitis. Eur Rev Med Pharmacol Sci 17:3178-3185. 


\section{Table $\mathbf{1}$ (on next page)}

Table 1 Primer and oligo sequences that were used in the study 
1

Table 1 Primer and oligo sequences that were used in the study

Primer name

GNLY

KLRD1

GZMB

PRF1

IL2RB

KLRB1

NKG7

CD247

GZMA

GAPDH
Primer sequence, $5^{\prime}$ to $3^{\prime}$

Forward: GTGTTCTGATTGATCCACAGTG Reverse: CATGTTTATTGAGTTTTGCGGC

Forward: CTACAGGACCTGTCTGACGATA Reverse: CAGCATTGGAAACACTTCTCTG

Forward: GTGAACAGAAAACTTGGAACGA Reverse: ATAGATACTGGGAGAGTGCAGA

Forward: GAAAGTGCGAATCTGACTTACG Reverse: TTGTTTCGTCCATAGGAGACAA

Forward: GCTATCGTTAGTGCTAGTGGAT Reverse: ATCTGTCTGATGCGTATCCAAT Forward: CTGAGATCTCGCCACTAGAAG Reverse: GGAAGAAGAAGTAACCCTGGTT

Forward: AATTTGCCCTGAAACTTAGCTG Reverse: GGATGTCACTGAAACACTCAAC Forward: GATGTTCTGCCTGATTGCTTTG Reverse: GGACAAGGACAAGAGAGATGG

Forward: ATAACGAGCTCAATCTAGGACG Reverse: CTGTACTGAGACCCTGGTAAAG Forward: GCGAAGGTGACCTTAAACTTTT Reverse: TGACTTCTCTCAGAGTATCGGA Forward: GGAGCGAGATCCCTCCAAAAT Reverse: GGCTGTTGTCATACTTCTCATGG- 


\section{Table 2 (on next page)}

Table 2 GO analysis results of DEGs (top 15 terms of BP category were listed). "Count" means how many DEGs are involved. 
Table 2 GO analysis results of DEGs (top 15 terms of BP category were listed). "Count" means how many DEGs are involved.

\begin{tabular}{cccc} 
ID & Description & p.adjust & Count \\
\hline GO:0043122 & regulation of I-kappaB kinase/NF-kappaB signaling & $3.00 \mathrm{E}-05$ & 17 \\
GO:0007249 & I-kappaB kinase/NF-kappaB signaling & $3.00 \mathrm{E}-05$ & 18 \\
GO:0043123 & positive regulation of I-kappaB kinase/NF-kappaB signaling & $3.00 \mathrm{E}-05$ & 15 \\
GO:0042119 & neutrophil activation & $5.63 \mathrm{E}-05$ & 24 \\
GO:0043312 & neutrophil degranulation & $9.15 \mathrm{E}-05$ & 23 \\
GO:0002228 & natural killer cell mediated immunity & $9.15 \mathrm{E}-05$ & 9 \\
GO:0002283 & neutrophil activation involved in immune response & $9.15 \mathrm{E}-05$ & 23 \\
GO:0002446 & neutrophil mediated immunity & 0.000119 & 23 \\
GO:0001819 & positive regulation of cytokine production & 0.000121 & 22 \\
GO:0031331 & positive regulation of cellular catabolic process & 0.000157 & 19 \\
GO:0009896 & positive regulation of catabolic process & 0.000391 & 20 \\
GO:0051091 & positive regulation of DNA-binding transcription factor activity & 0.000734 & 15 \\
GO:0006914 & autophagy & 0.000883 & 21 \\
GO:0061919 & process utilizing autophagic mechanism & 0.000883 & 21 \\
GO:0002237 & response to molecule of bacterial origin & 0.000917 & 17 \\
\hline
\end{tabular}

2 


\section{Table 3 (on next page)}

Table 3 KEGG analysis results of DEGs (top 10 pathways were listed). "Count" means how many DEGs are involved. 


\begin{tabular}{|c|c|c|c|}
\hline ID & Description & p.adjust & Count \\
\hline hsa04145 & Phagosome & 0.000184 & 14 \\
\hline hsa05140 & Leishmaniasis & 0.001378 & 9 \\
\hline hsa04640 & Hematopoietic cell lineage & 0.006969 & 9 \\
\hline hsa04650 & Natural killer cell mediated cytotoxicity & 0.009257 & 10 \\
\hline hsa04217 & Necroptosis & 0.034964 & 10 \\
\hline hsa04210 & Apoptosis & 0.034964 & 9 \\
\hline hsa05332 & Graft-versus-host disease & 0.034964 & 5 \\
\hline hsa05221 & Acute myeloid leukemia & 0.041801 & 6 \\
\hline hsa04658 & Th1 and Th2 cell differentiation & 0.041801 & 7 \\
\hline hsa04380 & Osteoclast differentiation & 0.050315 & 8 \\
\hline
\end{tabular}




\section{Table 4 (on next page)}

Table 4 Significantly enriched KEGG pathways for hub genes. "Count" means how many hub genes are involved. 
1

\begin{tabular}{|c|c|c|c|}
\hline ID & Description & p.adjust & Count \\
\hline hsa04650 & Natural killer cell mediated cytotoxicity & $5.58 \mathrm{E}-05$ & 4 \\
\hline hsa05332 & Graft-versus-host disease & 5.64E-05 & 3 \\
\hline hsa 05330 & Allograft rejection & 0.003575 & 2 \\
\hline hsa04940 & Type I diabetes mellitus & 0.003575 & 2 \\
\hline hsa05320 & Autoimmune thyroid disease & 0.004346 & 2 \\
\hline hsa04658 & Th1 and Th2 cell differentiation & 0.010825 & 2 \\
\hline hsa04659 & Th17 cell differentiation & 0.012492 & 2 \\
\hline hsa04210 & Apoptosis & 0.017483 & 2 \\
\hline hsa05202 & Transcriptional misregulation in cancer & 0.030326 & 2 \\
\hline
\end{tabular}

2 


\section{Figure 1}

Figure 1 Visualization of differentially expressed genes (DEGs).

(A) DEGs screened by threshold (adjusted $p$ value $<0.05$ and $|\log F C|>0.2$ ) were presented

by volcano plot. (B) Heatmap showed the expression of top 50 upregulated and downregulated genes ordered by adjusted $p$-value. Red indicated that the expression of genes was relatively upregulated, and the blue indicated the expression of genes was relatively downregulated. 
A

Volcano
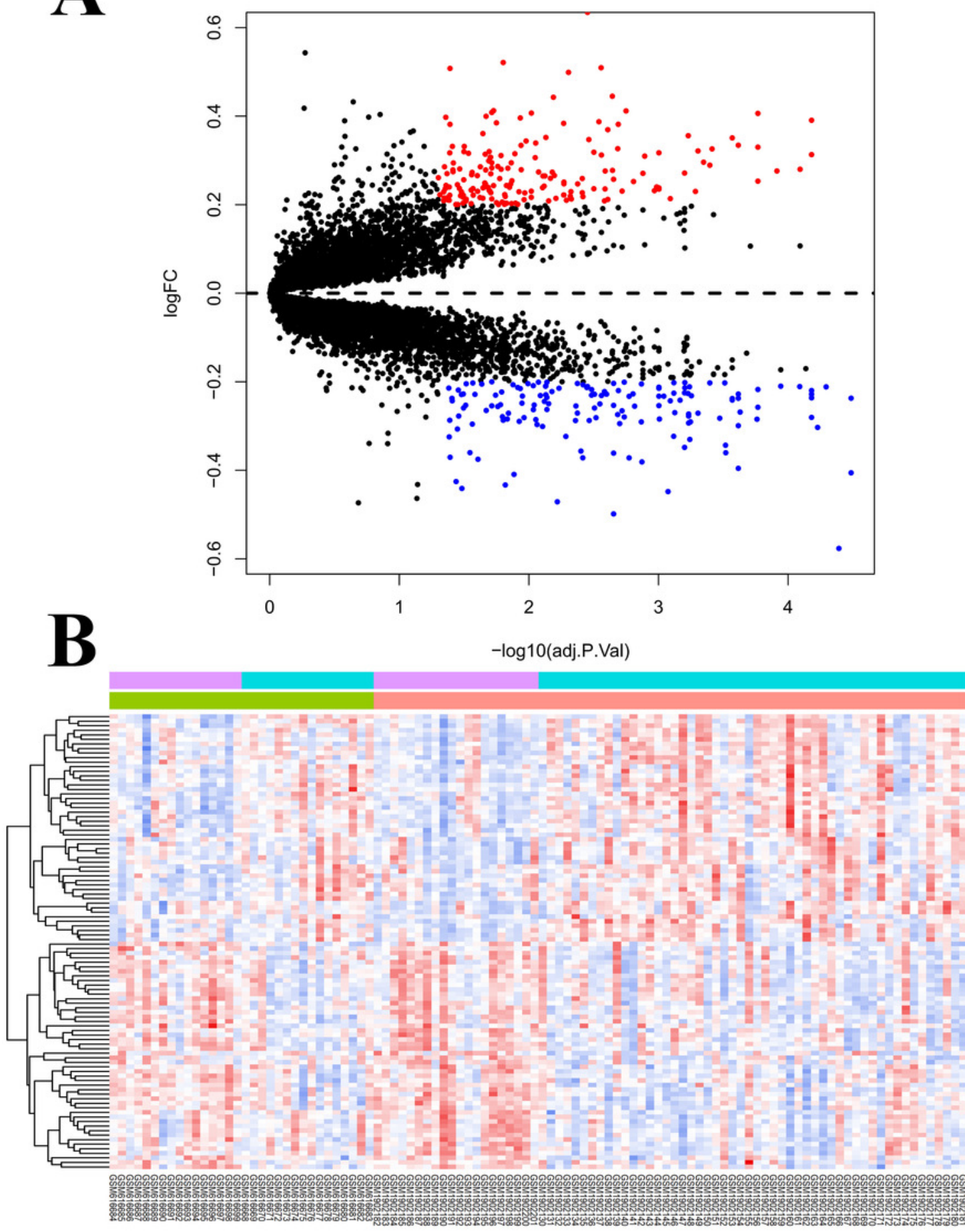

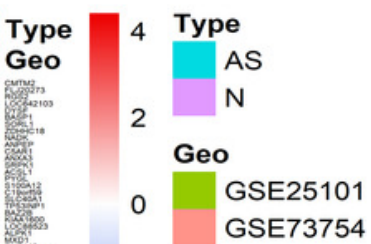
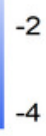
Figure 2

Figure 2: Results of functional enrichment analysis.

(A) GO analysis results of DEGs, top 15 terms in BP category were listed. (B) The top 9 pathways of KEGG analysis (According to the adjusted P value).
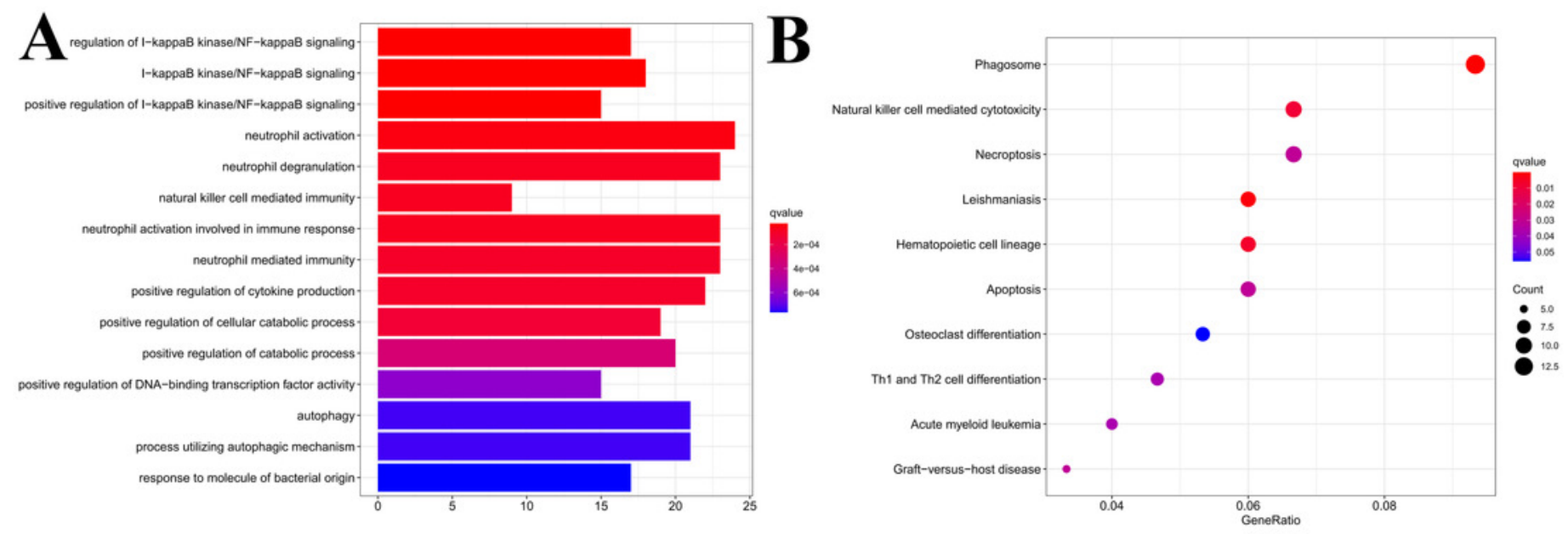
Figure 3

Figure 3: GSEA pathway enrichment analysis.

Pathway with FDR $<0.05$ were considered to be significance.

- Autophagy - animal - Complement and coagulation cascades - Leukocyte transendothelial migration - Osteoclast differentiation

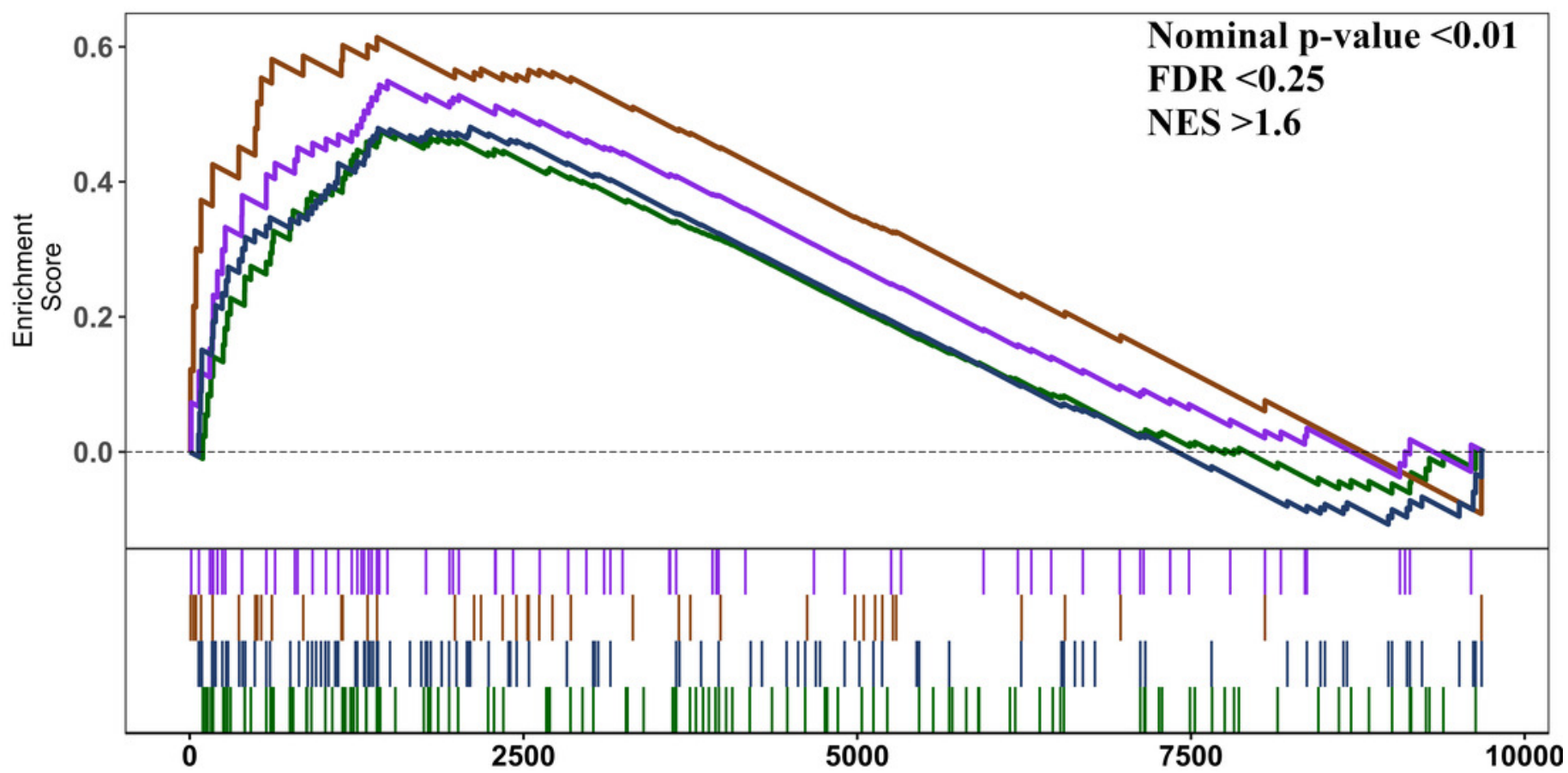




\section{Figure 4}

Figure 4: PPI network construction and module analysis.

(A) The PPI network of DEGs was constructed in Cytoscape, Nodes represent proteins and edges represent interactions between two proteins. (B) The most significant module was obtained by MCODE plug-in. Red nodes represent upregulated DEGs and blue nodes represent downregulated DEGs. The diameters of nodes were positively correlated with their connectivity degree. (C) Hub genes selected by calculating the MCC scores of cytoHubba plugin. 


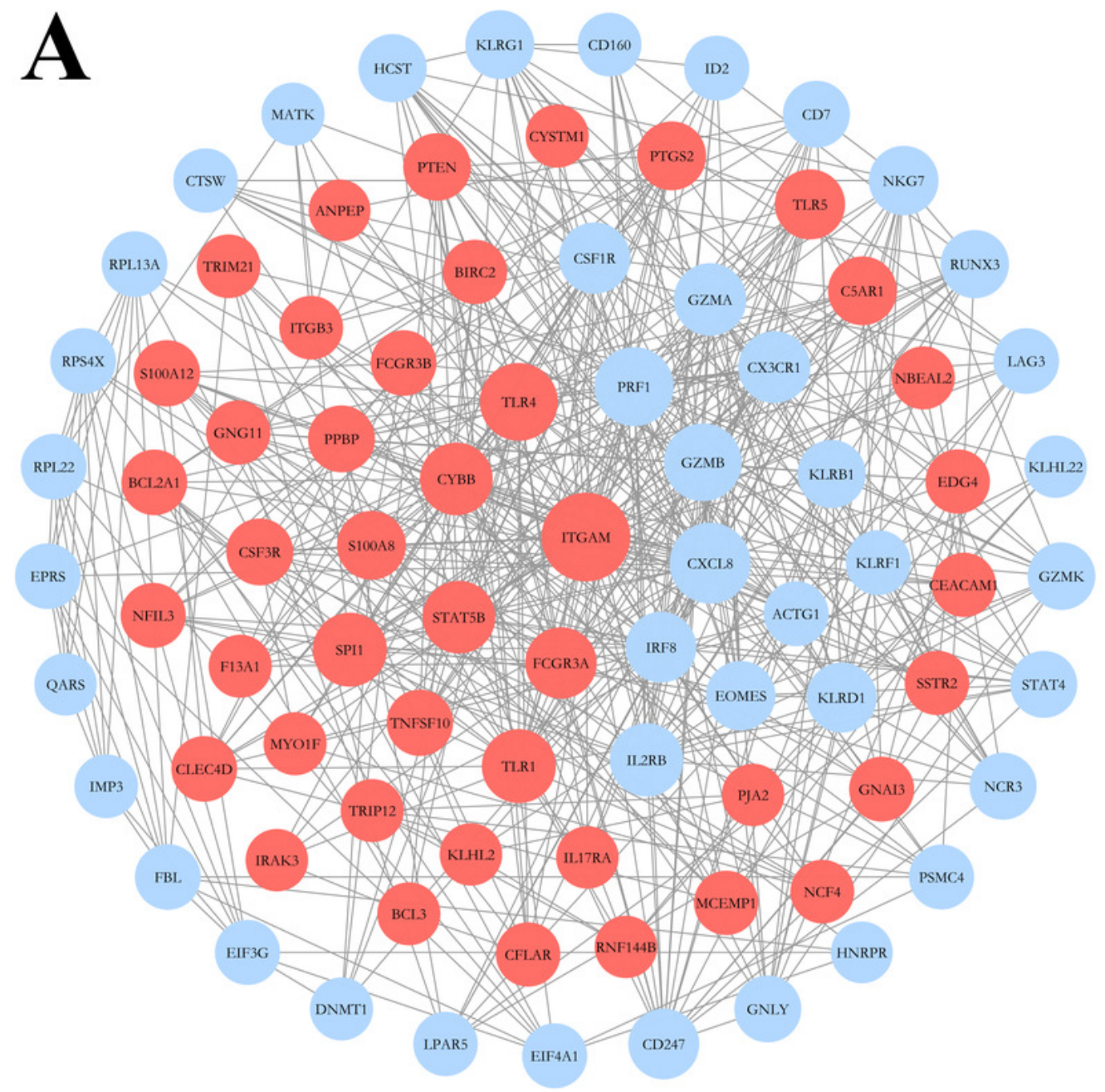

B

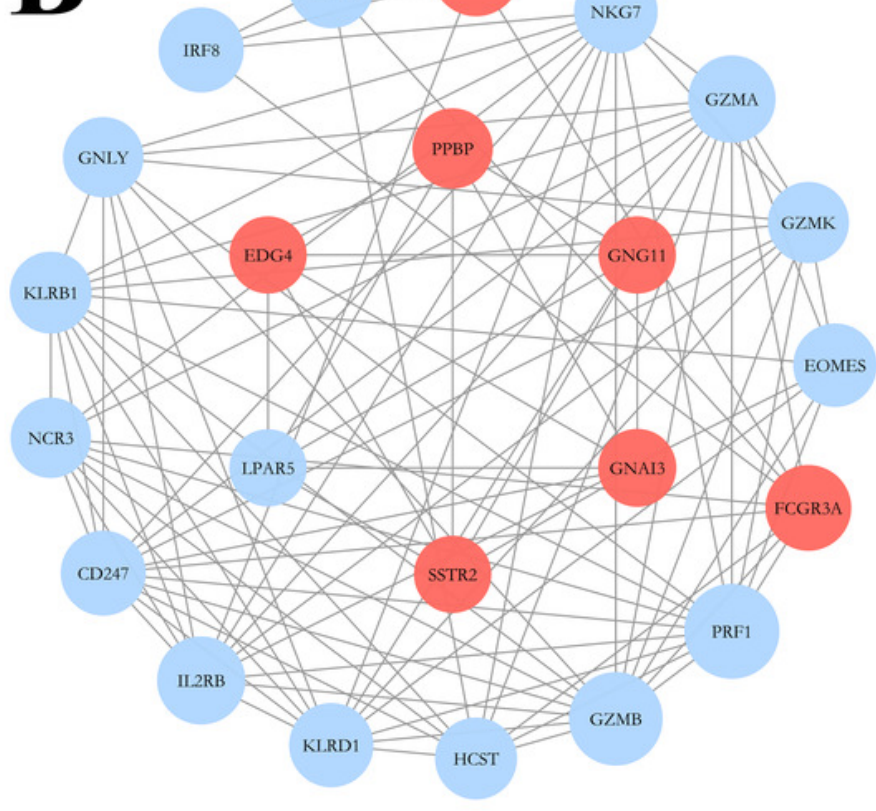

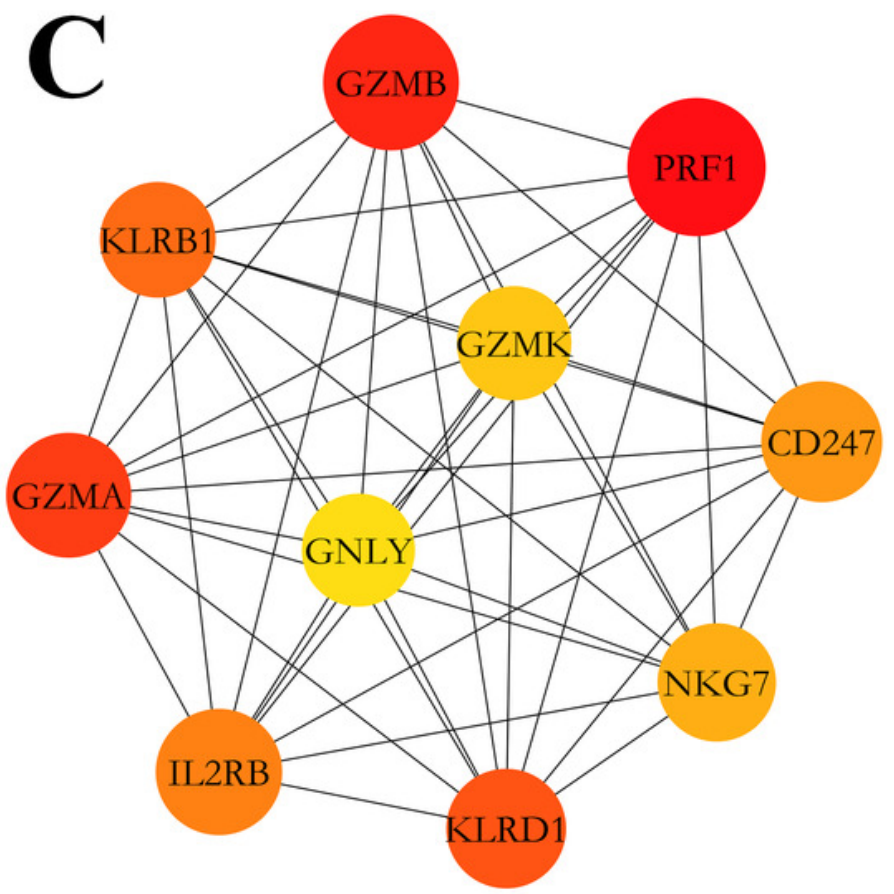




\section{Figure 5}

Figure 5: The profiles of immune cell subtype distribution pattern in GSE25101 and GSE73754 cohort.

(A) The bar plot visualizing the relative percent of 22 immune cell in each sample. (B) Heatmap of the 22 immune cell proportions in each sample. (C) Correlation heatmap of all 22 immune cells. (D) Violin plot of all 22 immune cells differentially infiltrated fraction. 


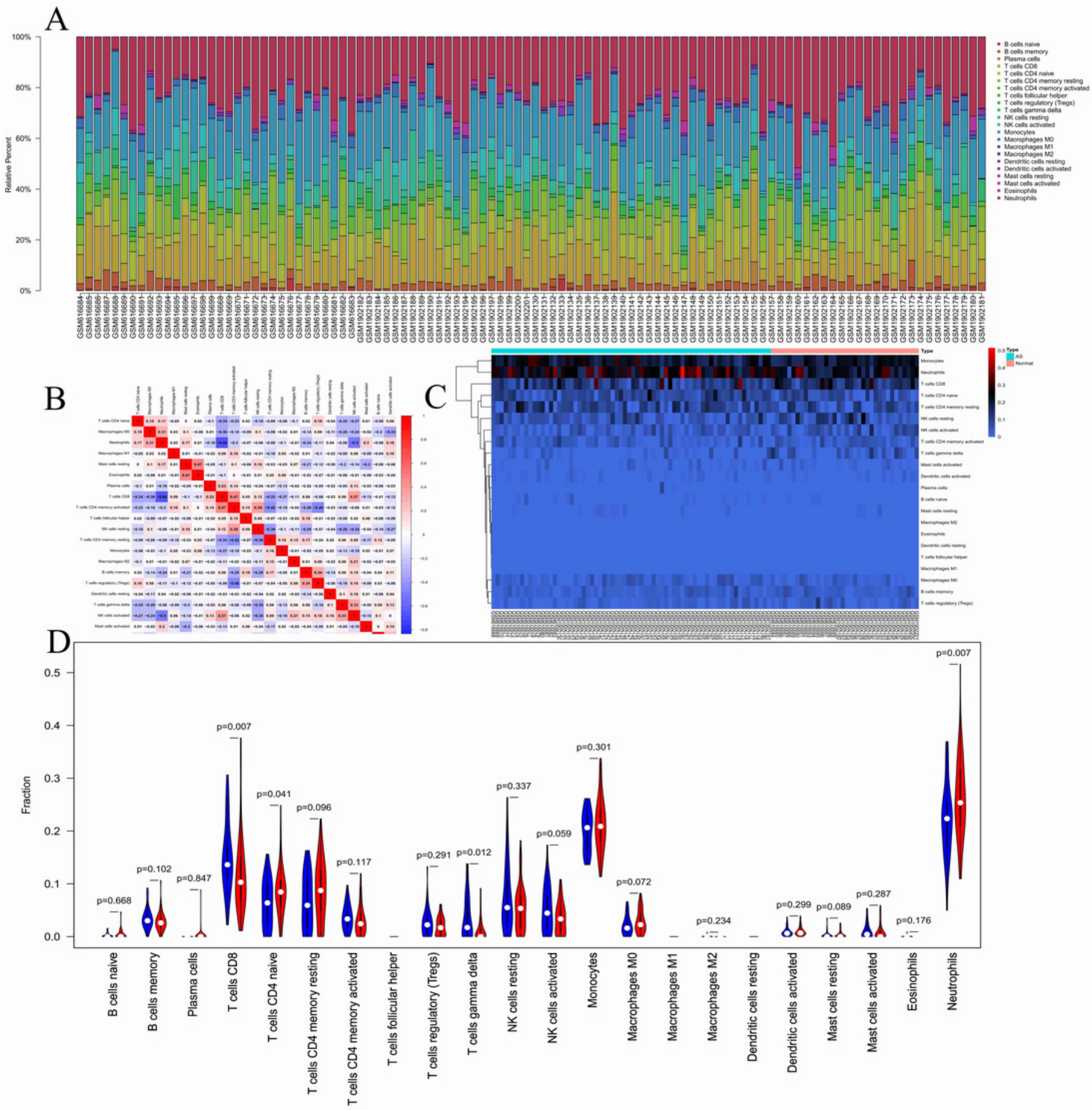


Figure 6

Figure 6 RT-PCR validation of the hub gene between AS and normal controls.

Including (A)CD247, (B) GNLY, (C) GZMA, (D) GZMB, (E) GZMK, (F) IL2RB, (G) KLRB1, (H) KLRD1, (I) NKG7 and (J) PRF1. (* P $<0.01)$.

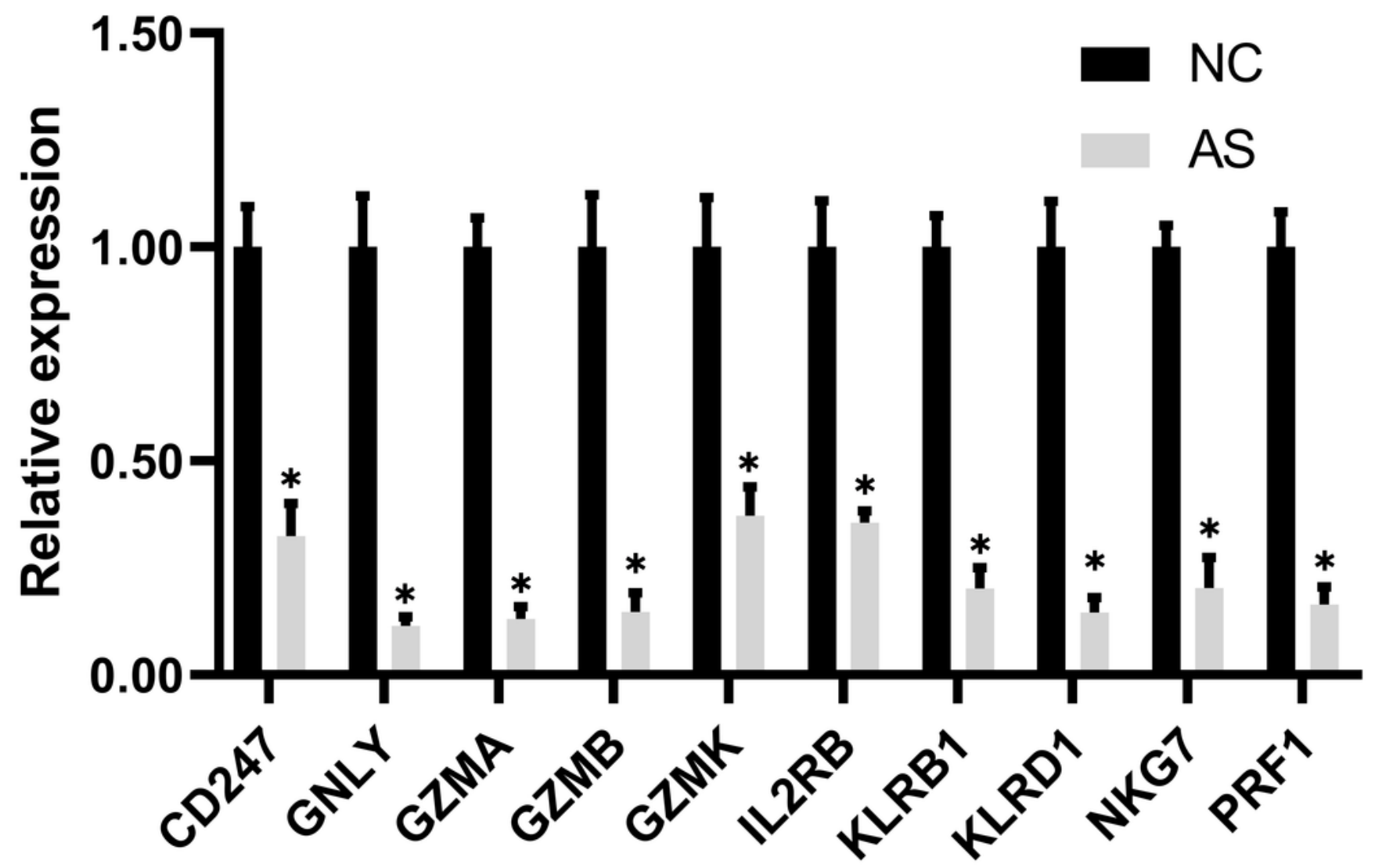


Figure 7

Figure 7: ELISA validation of the protein expression.

ELISA analysis of GZMA, GNLY and PRF1 expression in serum between AS and normal controls. $(* P<0.05)$.

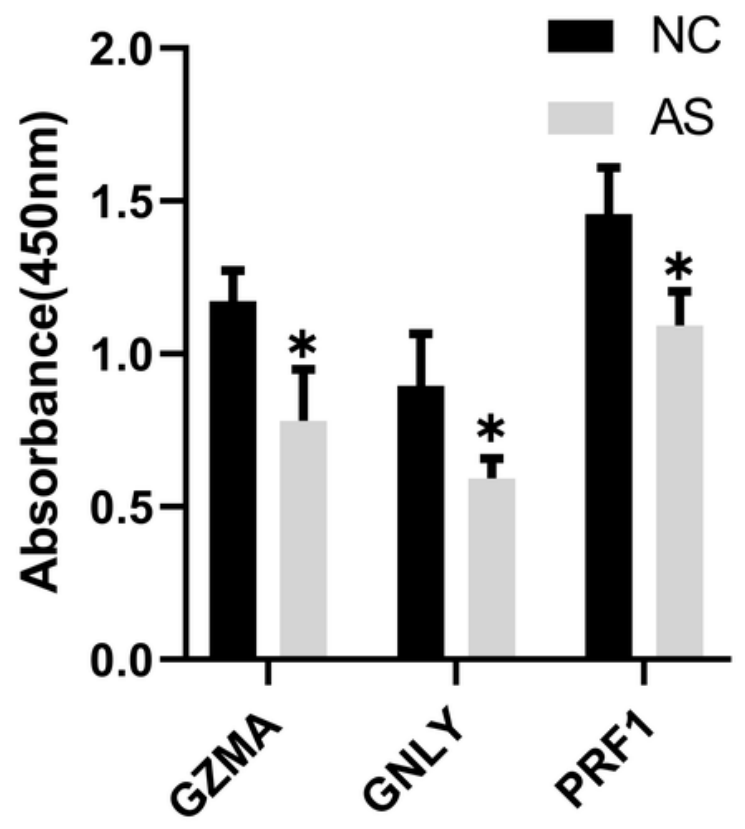


Figure 8

Figure 8: Validation of candidate hub genes by ROC curve analysis.

Among the 10 genes screened out by cytoHubba plug-in, nine genes with AUC more than 0.70 were considered as hub genes of AS. 

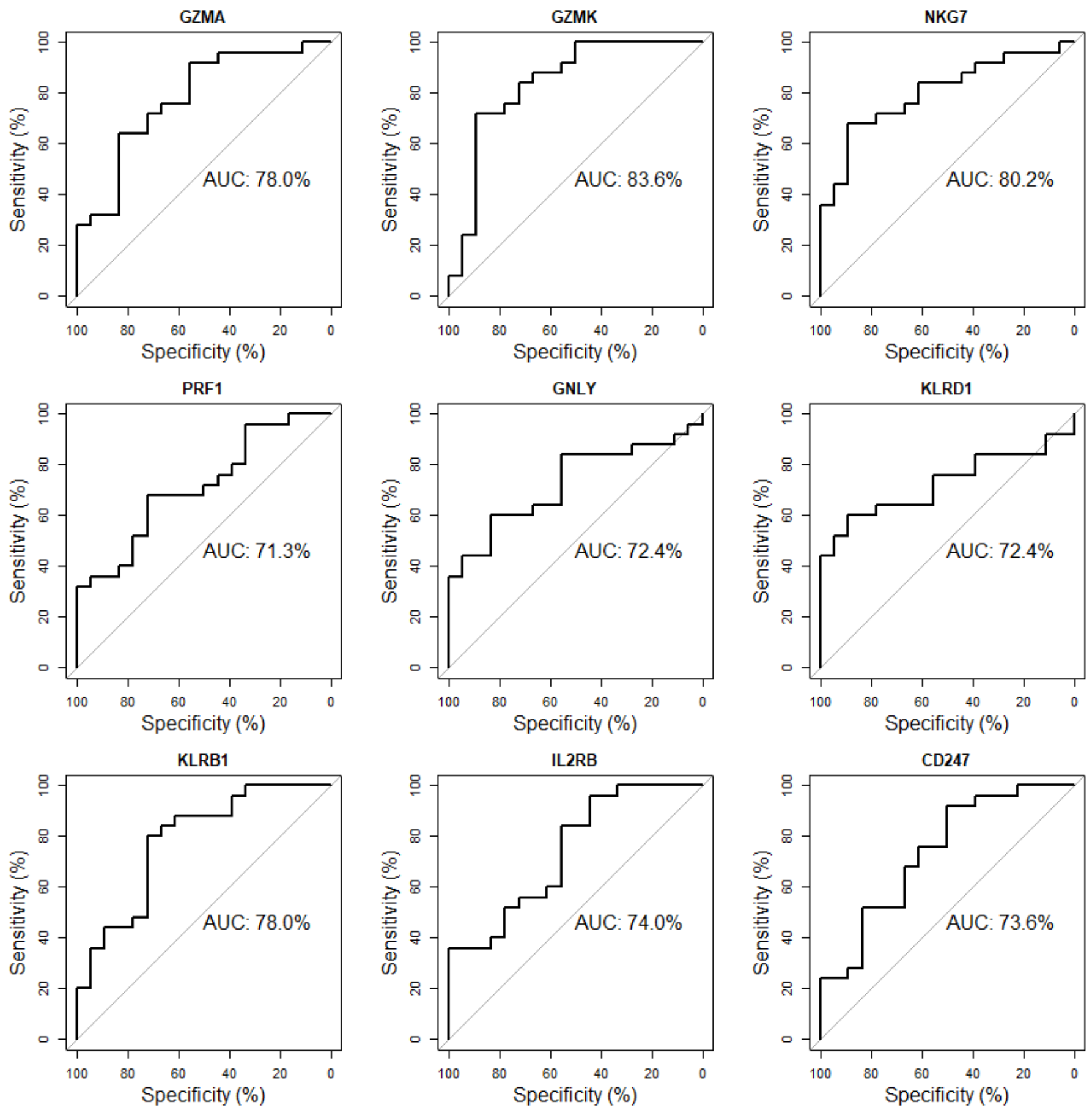